Vilma Sousa Santana ${ }^{1}$

Gustavo Ribeiro de Araújo ${ }^{1}$

Jônatas Silva do Espírito-Santo ${ }^{1}$

José Bouzas de Araújo-Filho ${ }^{1}$

Jorge Iriart ${ }^{1}$

\section{A utilização de serviços de saúde por acidentados de trabalho}

\author{
Health services utilization by occupational injured workers
}

${ }^{1}$ Programa Integrado de Saúde Ambiental e do Trabalhador (PISAT), Instituto de Saúde Coletiva (ISC), Universidade Federal da Bahia (UFBA), Salvador-BA.

Fontes de financiamento: Ministério de Ciência e Tecnologia, Conselho Nacional de Desenvolvimento Científico e Tecnológico CNPq/Proc. 521226-98-8, Projeto Nordeste de Pesquisa e PIBIC/ UFBA; Secretaria de Planejamento do Estado da Bahia, Superintendência de Apoio ao Desenvolvimento Científico e Tecnológico (CADCT) nº68/2000. Ministério da Saúde, Área Técnica da Saúde do Trabalhador.

\section{Resumo}

Neste estudo descrevem-se as características da utilização de serviços de saúde por trabalhadores que sofreram acidentes de trabalho. Os dados provêm das três primeiras fases de um estudo de coorte de base comunitária sobre saúde e trabalho, iniciado no ano 2000, e conduzido com todos os trabalhadores de 2.512 famílias selecionadas por amostragem aleatória de conglomerado, de estágio único, residentes na cidade de Salvador, Bahia. Entrevistas individuais foram empregadas para a coleta de dados. Nesta pesquisa analisaram-se os trabalhadores $(\mathrm{n}=628)$ que referiram ter sofrido acidente de trabalho nos 12 meses antes da entrevista. Observou-se que cerca de metade dos casos de acidentes recebeu primeiros socorros e tratamento médico. A maioria (71\%) foi atendida em unidades do SUS. Cerca de 15\% tiveram o atendimento pago por planos de saúde privados. Observou-se também que a maioria referiu alta satisfação com o atendimento, tanto em serviços públicos como privados. Entre os usuários do SUS, predominaram os trabalhadores sem carteira assinada, mas trabalhadores segurados também utilizaram os serviços públicos em sua maioria. Concluiu-se que o SUS tem expressiva participação no atendimento de acidentados do trabalho, independentemente da condição de cobertura por planos de saúde.

Palavras-chaves: acidentes de trabalho, acidentes ocupacionais, acesso e utilização de serviços, atenção à saúde de acidentados do trabalho.

\begin{abstract}
This study describes the characteristics of health services utilization by workers reporting work-related injuries. Data comes from the three first phases of a community-based cohort study about health and work that started in the year 2000, carried out with all workers from 2,512 families living in the city of Salvador, Bahia, that were selected by one-stage cluster area random sampling. Individual interviews were used for data collection. In this study, cases of work injuries reported during the 12 months before the interview $(n=628)$ are analyzed. The majority (71.0\%) of injured workers received medical treatment in facilities from the Unified Health System, SUS, a public health care system of universal coverage. Around 15\% received treatment from private health insurance plans. Among SUS customers most workers have no formal job contracts, although insured workers also utilized public health care services. In sum, the SUS has expressive participation in the health care of injured workers independently of their entitlement for private health care.
\end{abstract}

Keywords: work injuries, occupational injuries, health care access and utilization, health care for injured workers. 


\section{Introdução}

No Brasil, os serviços de saúde se organizam em torno do Sistema Único de Saúde (SUS), de cobertura universal, sob a responsabilidade do Estado com a participação da atenção suplementar a cargo de empresas privadas. Os serviços de saúde caracterizam-se também por diferenças marcantes no acesso e na utilização de serviços (PAIM, 2002). Como a saúde é importante fator de produtividade econômica, apesar da existência do SUS e de sua cobertura universal, algumas empresas fornecem planos de seguro-saúde para os seus trabalhadores, que permitem o acesso a serviços ambulatoriais, hospitalares ou assistência domiciliar (homecare) com cobertura total ou parcial das despesas, algumas vezes com uma contribuição do trabalhador no pagamento das mensalidades. $\mathrm{O}$ acesso a esses planos de saúde é restrito a trabalhadores formais, com contrato formal de trabalho na empresa. Algumas empresas de médio ou grande porte oferecem serviços ambulatoriais ou de pronto atendimento nas suas próprias instalações. Para os trabalhadores informais, comuns em empresas não registradas ou na economia informal, e também para os autônomos ou conta-própria, a cobertura por serviços de saúde se dá pelo SUS ou com o pagamento do próprio bolso, seja por meio de planos privados individuais de saúde ou consulta e procedimentos específicos. Em geral, esses serviços são empregados para agravos ou enfermidades, independentemente da sua natureza, se ocupacional ou não (BEDRIKOW et al., 1997).

Os acidentes ocupacionais são responsáveis pelo maior número de mortes e incapacidades graves causados pelo trabalho em todo o mundo, embora muitos países não diferenciem as estatísticas dos acidentes em relação às enfermidades ocupacionais (TAKALA, 1999). Em uma revisão dos estudos sobre acidentes de trabalho no Brasil, verificou-se que a mortalidade anual por acidentes de trabalho é estimada em 13,2/100.000 trabalhadores segurados e que a incidência cumulativa anual para os acidentes não-fatais, com base em dados da população geral, varia entre $3 \%$ a $6 \%$.

Esses agravos têm uma expressiva participação nos custos com a saúde no país. Com dados do Instituto Nacional de Seguridade Social (INSS), sabe-se que os acidentes de trabalho representam mais da metade dos benefícios pagos pela Previdência Social $(62,8 \%)$ para afastamentos do trabalho por enfermidades e agravos ocupacionais no Brasil (SANTANA et al., 2006). Infelizmente não é conhecido o montante dos custos com o tratamento de acidentes do trabalho, embora, com dados das Autorizações de Internações Hospitalares (AIH), os agravos por causas externas alcançaram o $1^{\circ}$ lugar dentre as despesas hospitalares no SUS (BRASIL, 2002).

Pessoas acometidas por acidentes de trabalho constituem uma parte significativa dos atendimentos em serviços médicos, especialmente nos serviços de emergência. Com dados desses serviços, em Salvador, Bahia, Conceição et al. (2003) verificaram que $31,8 \%$ dos atendimentos devidos a causas externas eram acidentes de trabalho. Analisando-se dados de toda a rede municipal, Vilela et al. (2001) estimaram que, em Piracicaba, cerca de $59,7 \%$ dos acidentes e doenças do trabalho eram atendidos diretamente pelo SUS, enquanto que a rede privada e filantrópica se responsabilizava por aproximadamente 40,3\% dos casos. Em estudo com metodologia parecida, com dados de um estudo de base populacional realizado em Botucatu, São Paulo, Cordeiro et al. (2005) estimaram que a maioria $(80,7 \%)$ dos trabalhadores recebeu atendimento médico em unidades do SUS.

São poucas as informações disponíveis, de base populacional, sobre o acesso e a utilização de serviços de saúde por parte de trabalhadores acidentados, apesar da sua importância. O conhecimento acerca dessa realidade é especialmente relevante para o processo de implantação da Rede Nacional de Atenção à Saúde do Trabalhador (RENAST) e a participação do Programa de Saúde da Família na atenção à saúde do trabalhador. Este estudo pretende reduzir essa lacuna, descrevendo as características da utilização de serviços de saúde por trabalhadores que sofreram acidentes de trabalho com base nos dados de um estudo de coorte de base populacional. 


\section{Métodos}

Os dados analisados compreendem os casos de acidentes de trabalho identificados nas três primeiras fases de um estudo de coorte prospectivo, de base comunitária, iniciado no ano 2000 na cidade do Salvador, capital do estado da Bahia. Este estudo vem sendo conduzido com todos os membros de 2.512 famílias selecionadas por amostragem aleatória de conglomerado, por estágio único, tendo sido entrevistados todos os trabalhadores em 2000 e 2004. Em 2002, apenas uma subamostra foi re-entrevistada. A população do estudo compreende todos os casos de acidentados que tinham idade entre 18 e 65 anos, identificados em cada uma das fases mencionadas, respectivamente, 258, 38 e 368, com um total de 628 registros. Todas as residências selecionadas foram visitadas. Após a obtenção de consentimento informado, dados sociodemográficos de cada um dos membros da família foram registrados. Para os que se identificavam como trabalhadores remunerados, realizaram-se entrevistas individuais para o registro de dados sobre características ocupacionais e hábitos de vida; e para os acidentes, características das lesões, condições de ocorrência, tratamento recebido, duração do afastamento do trabalho e do tratamento e escore de satisfação com o atendimento, dentre outras informações (SANTANA et al., 2003).

Considerou-se como acidente de trabalho "qualquer dano infligido ao corpo por transferência de energia durante o trabalho, ou deslocamento até o local do trabalho que envolvesse uma curta duração entre exposição e efeitos identificáveis após a ocorrência do evento/ circunstância” (HAGBERG et al. 1997). Para garantir que todos os casos seriam reconhecidos independentemente do vínculo com o trabalho, não se formulava pergunta direta sobre acidente de trabalho, mas sim sobre acidentes em geral. A vinculação com o trabalho era identificada com base nas circunstâncias descritas na narrativa feita pelo próprio trabalhador. Os acidentes foram classificados em "de trajeto" ou "típicos". Para a descrição da população, utilizaram-se variáveis sociodemográficas, como sexo, grupo de idade (18-30, 31-45, 41-65), cor da pele (negros e não negros), escolaridade (baixo = até o nível elementar; médio = elementar completo; e alto = superior completo ou incompleto). A variável "nível socioeconômico" foi criada a partir do número de bens da família ou equipamentos disponíveis no domicílio, especificamente: automóvel, computador, máquina de lavar, videocassete, tocadiscos a laser, microondas, máquina de lavar louça, telefone e casa de praia. A soma dos itens foi categorizada em: baixo $=$ menos de 3 itens; médio $=3$ a 5 itens; e alto = acima de 5 itens. O tipo de vinculação ao mercado de trabalho foi definido como informal e formal para os contratados formalmente, com carteira de trabalho assinada. As variáveis relativas à utilização de serviços foram: recebimento de primeiros socorros, o local onde recebeu esse tipo de atendimento, se recebeu atendimento médico após os primeiros socorros, duração do tratamento em dias e a instituição/fonte pagadora. A satisfação com o atendimento recebido foi registrada mediante notas de 1 a 10 atribuídas pelos entrevistados e obtidas com a marcação feita pelo próprio trabalhador em uma escala com as respectivas indicações. Essas notas foram analisadas em três grupos: baixo = abaixo de 7,0; médio = entre 7,1 e 8,0; e alto $=$ entre 8,1 e 10).

Freqüências simples e relativas em porcentuais foram estimadas e inferências estatísticas realizadas com o teste do Qui Quadrado de Pearson, quando indicado. As análises foram realizadas utilizando o software de análise de dados estatísticos SAS, versão 9.0. O protocolo de pesquisa foi submetido e aprovado por uma Comissão de Ética em Pesquisa da Universidade Federal da Bahia, tendo sido atendidas todas as recomendações legais. O anonimato e a confidencialidade das informações foram mantidas nos registros dos dados e nas publicações. Toda a equipe da pesquisa foi instruída sobre os dispositivos e as responsabilidades éticas do estudo. 


\section{Resultados}

Dos 628 trabalhadores que referiram acidentes de trabalho ao longo do seguimento do estudo, predominaram as mulheres (64\%), pessoas mais jovens entre 18-30 anos $(41,6 \%)$, negros $(66,4 \%)$, pessoas de baixa escolaridade $(51,4 \%)$, de mais baixo nível socioeconômico (NSE) $(55,1 \%)$ e trabalhadores com contrato informal de trabalho (54,3\%) (Tabela 1). A maioria dos acidentes (89\%) foi classificada como típico e apenas $11 \%$ ocorreram no trajeto.

Apenas metade dos casos $(49,5 \%, \mathrm{n}=$ 311) recebeu primeiros socorros imediatamente após o acidente (Tabela 2). Destes, a maioria foi levada para serviços de emergência médica $(47,6 \%, n=148)$, enquanto uma parte substancial dos casos referiu ter recebido tratamento médico após os primeiros socorros $(48,9 \%, n=152)$. Este último tratamento teve uma duração média de duas semanas e foi, em sua maioria, considerado altamente satisfatório. Aproximadamente 51,3\% atribuíram notas entre 8,1 e 10, sendo que o atendimento se concentrou em unidades do SUS (71\%). Planos de saúde foram responsáveis por 15,1\% ( $\mathrm{n}=$ 23), enquanto a empresa empregadora participou com apenas $4 \%$ dos tratamentos (n $=6)$. Cerca de 5,3\% (n=8) dos trabalhadores pagaram suas próprias despesas com o tratamento.

Na Tabela 3 observa-se que pessoas mais jovens e as que sofreram acidentes de trajeto receberam mais comumente tratamento médico do que as de mais idade ou que referiram acidente típico, respectivamente, $p<0,05$. Não houve diferenças no recebimento de tratamento por sexo, escolaridade, cor da pele, nível socioeconômico e informalidade do contrato de trabalho. $\mathrm{O}$ alto grau de satisfação com o tratamento foi menor entre os trabalhadores informais $(38,5 \%)$ do que entre os contratados formais $(60,6 \%, p<0,001)$. Nenhuma das outras diferenças relativas ao grau de satisfação foi estatisticamente significante.

Tabela 1 Características socioeconômicas dos acidentados entrevistados em Salvador/BA no período de 2001 e 2004

\begin{tabular}{|c|c|c|}
\hline Variáveis & $N=628$ & $\%$ \\
\hline \multicolumn{3}{|l|}{ Sexo } \\
\hline Feminino & 402 & 64,0 \\
\hline Masculino & 226 & 36,0 \\
\hline \multicolumn{3}{|l|}{ Idade } \\
\hline $18-30$ & 261 & 41,6 \\
\hline $31-40$ & 162 & 25,8 \\
\hline $41-65$ & 205 & 32,6 \\
\hline \multicolumn{3}{|l|}{ Escolaridade } \\
\hline Baixa & 323 & 51,4 \\
\hline Média & 269 & 42,8 \\
\hline Alta & 36 & 5,8 \\
\hline \multicolumn{3}{|l|}{ Cor da pele } \\
\hline Negra & 417 & 66,4 \\
\hline Não negra & 211 & 33,6 \\
\hline \multicolumn{3}{|c|}{ Nível socioeconômico } \\
\hline Baixo & 346 & 55,1 \\
\hline Médio & 191 & 30,4 \\
\hline Alto & 91 & 14,5 \\
\hline \multicolumn{3}{|c|}{ Trabalho informal } \\
\hline Sim & 248 & 54,3 \\
\hline Não & 209 & 45,7 \\
\hline \multicolumn{3}{|l|}{ Tipo do acidente } \\
\hline Típico & 559 & 89,0 \\
\hline Trajeto & 69 & 11,0 \\
\hline
\end{tabular}


A mais comum instituição prestadora/ financiadora de atendimento médico para os indivíduos que sofreram acidente de trabalho foi o SUS (Tabela 4). A proporção de casos atendidos/financiados pelo SUS variou de $50 \%$, estimada entre os trabalhadores de alto nível socioeconômico, a $82,7 \%$ entre os trabalhadores sem contrato formal de trabalho. Não houve variações significativas na distribuição das proporções de usuários do SUS, exceto para o trabalho informal, com maior proporção de clientes do SUS entre os trabalhadores sem contrato quando comparados aos formais $(\mathrm{p}<0,001)$. Embora a alta satisfação com o tratamento recebido tenha predominado para os usuários do SUS (48,2\%) e de outras instituições/fontes de financiamento, a proporção foi maior entre estes últimos (60,5\%), que também ficou com a menor cifra de baixa satisfação $(20,9 \%)$, conforme Tabela 5.

Tabela 2 Utilização de serviços de saúde pelos trabalhadores acidentados entrevistados em Salvador/BA no período de 2001 e 2004

\begin{tabular}{|c|c|c|}
\hline Variáveis & $N$ & $\%$ \\
\hline \multicolumn{3}{|l|}{ Você precisou de primeiros socorros? $(\mathrm{N}=628)$} \\
\hline Sim & 311 & 49,5 \\
\hline Não & 317 & 50,5 \\
\hline \multicolumn{3}{|l|}{ Onde recebeu os primeiros socorros? $(\mathrm{N}=311)$} \\
\hline Em casa & 48 & 15,4 \\
\hline No local de trabalho por colegas & 31 & 10,0 \\
\hline No serviço médico da empresa & 24 & 7,7 \\
\hline Serviço de emergência & 148 & 47,6 \\
\hline Serviço médico ambulatorial & 48 & 15,4 \\
\hline Ambulância & 1 & 0,3 \\
\hline Outros & 11 & 3,6 \\
\hline \multicolumn{3}{|c|}{ Após esse atendimento, recebeu tratamento médico? ( $\mathrm{N}=311)$} \\
\hline Sim & 152 & 48,9 \\
\hline Não & 152 & 48,9 \\
\hline Não informou & 7 & 2,2 \\
\hline \multicolumn{3}{|c|}{ Duração do tratamento em dias $(\mathrm{N}=152)$} \\
\hline $0-6$ & 45 & 29,6 \\
\hline $7-15$ & 52 & 34,2 \\
\hline$>15$ & 37 & 24,3 \\
\hline Não informou & 18 & 11,8 \\
\hline \multicolumn{3}{|c|}{ Nota atribuída ao tratamento $(\mathrm{N}=152)$} \\
\hline Baixa $(0-7,0)$ & 43 & 28,3 \\
\hline Média $(7,1-8,0)$ & 31 & 20,4 \\
\hline Alta $(8,1-10)$ & 78 & 51,3 \\
\hline \multicolumn{3}{|c|}{ Instituição/financiamento do tratamento ( $N=152)$} \\
\hline SUS & 108 & 71,0 \\
\hline Empresa empregadora & 6 & 4,0 \\
\hline Plano de saúde privado & 23 & 15,1 \\
\hline Próprio bolso & 8 & 5,3 \\
\hline Seguro acidente (privado) & 1 & 0,8 \\
\hline Outros & 5 & 3,3 \\
\hline Não informou & 1 & 0,8 \\
\hline
\end{tabular}


Tabela 3 Características dos trabalhadores acidentados entrevistados em Salvador/BA no período de 2001 e 2004 que receberam tratamento e a satisfação destes com o atendimento recebido

\begin{tabular}{|c|c|c|c|c|c|c|c|c|}
\hline \multirow{3}{*}{ Variáveis } & \multicolumn{2}{|c|}{$\begin{array}{c}\text { Recebeu tratamento } \\
\text { médico }\end{array}$} & \multicolumn{6}{|c|}{ Grau de satisfação com o tratamento } \\
\hline & \multirow[t]{2}{*}{$N=304$} & \multirow[t]{2}{*}{$\%$} & \multicolumn{2}{|c|}{$0 a 7$} & \multicolumn{2}{|c|}{$8 a 9$} & \multicolumn{2}{|c|}{10} \\
\hline & & & $N$ & $\%$ & $N$ & $\%$ & $N$ & $\%$ \\
\hline \multicolumn{9}{|l|}{ Sexo } \\
\hline Feminino & 157 & 49,7 & 24 & 30,8 & 13 & 16,7 & 41 & 52,6 \\
\hline Masculino & 147 & 50,3 & 19 & 25,7 & 18 & 24,3 & 37 & 50,0 \\
\hline \multicolumn{9}{|l|}{ Idade } \\
\hline $18-30$ & 124 & $46,0^{*}$ & 16 & 28,1 & 14 & 24,6 & 27 & 47,4 \\
\hline $31-40$ & 81 & 44,4 & 11 & 30,6 & 5 & 13,9 & 20 & 55,6 \\
\hline $41-65$ & 99 & 36,2 & 16 & 27,1 & 12 & 20,3 & 31 & 52,5 \\
\hline \multicolumn{9}{|l|}{ Escolaridade } \\
\hline Baixa & 154 & 52,6 & 25 & 30,9 & 16 & 19,8 & 40 & 49,4 \\
\hline Média & 131 & 46,6 & 16 & 26,2 & 13 & 21,3 & 32 & 52,5 \\
\hline Alta & 19 & 52,6 & 2 & 20,0 & 2 & 20,0 & 6 & 60,0 \\
\hline \multicolumn{9}{|l|}{ Cor da pele } \\
\hline Negra & 189 & 51,9 & 27 & 27,6 & 22 & 22,5 & 49 & 50,0 \\
\hline Não negra & 115 & 47,0 & 16 & 29,6 & 9 & 16,7 & 29 & 53,7 \\
\hline \multicolumn{9}{|c|}{ Nível socioeconômico } \\
\hline Baixo & 161 & 50,4 & 23 & 28,8 & 15 & 18,8 & 42 & 52,5 \\
\hline Médio & 102 & 53,0 & 13 & 24,1 & 11 & 20,4 & 30 & 55,6 \\
\hline Alto & 41 & 38,7 & 7 & 38,9 & 5 & 27,8 & 6 & 33,3 \\
\hline \multicolumn{9}{|l|}{ Trabalho informal } \\
\hline Sim & 139 & 51,1 & 15 & 28,9 & 17 & 32,7 & 20 & $38,5^{* * *}$ \\
\hline Não & 110 & 47,3 & 19 & 26,8 & 9 & 12,7 & 43 & 60,6 \\
\hline \multicolumn{9}{|l|}{ Tipo do acidente } \\
\hline Típico & 259 & $47,9^{*}$ & 33 & 26,6 & 27 & 21,8 & 64 & 51,6 \\
\hline Trajeto & 45 & 62,6 & 10 & 35,7 & 4 & 14,3 & 14 & 50,0 \\
\hline
\end{tabular}

" $\mathrm{p}<0,10 ;{ }^{* * * *} \mathrm{p}<0,05 ;{ }^{* * * *} \mathrm{p}<0,001$ 
Tabela 4 Utilização e satisfação relatada pelos acidentados que receberam tratamento, de acordo com o nível socioeconômico

\begin{tabular}{|c|c|c|c|c|}
\hline \multirow{3}{*}{ Variáveis } & \multicolumn{4}{|c|}{ Instituição/financiamento } \\
\hline & \multicolumn{2}{|c|}{ SUS } & \multicolumn{2}{|c|}{ Outros } \\
\hline & $N$ & $\%$ & $N$ & $\%$ \\
\hline \multicolumn{5}{|l|}{ Sexo } \\
\hline Feminino & 56 & 72,7 & 21 & 27,3 \\
\hline Masculino & 52 & 70,3 & 22 & 29,7 \\
\hline \multicolumn{5}{|l|}{ Idade } \\
\hline $18-30$ & 56 & 75,0 & 14 & 25,0 \\
\hline $31-40$ & 26 & 72,2 & 10 & 27,8 \\
\hline $41-65$ & 40 & 67,8 & 19 & 32,2 \\
\hline \multicolumn{5}{|l|}{ Escolaridade } \\
\hline Baixa & 62 & 76,5 & 19 & 23,5 \\
\hline Média & 41 & 68,3 & 19 & 31,7 \\
\hline Alta & 5 & 50,0 & 5 & 50,0 \\
\hline \multicolumn{5}{|l|}{ Cor da pele* } \\
\hline Negra & 65 & 67,0 & 32 & 33,0 \\
\hline Não negra & 43 & 79,6 & 11 & 20,4 \\
\hline \multicolumn{5}{|l|}{ Nível socioeconômico } \\
\hline Baixo & 62 & 77,5 & 18 & 22,5 \\
\hline Médio & 36 & 66,7 & 18 & 33,3 \\
\hline Alto & 10 & 58,8 & 7 & 41,2 \\
\hline \multicolumn{5}{|l|}{ Trabalho informal"***** } \\
\hline Sim & 43 & 82,7 & 9 & 17,3 \\
\hline Não & 42 & 59,2 & 29 & 40,9 \\
\hline \multicolumn{5}{|l|}{ Tipo do acidente } \\
\hline Típico & 88 & 71,5 & 35 & 28,5 \\
\hline Trajeto & 20 & 71,4 & 8 & 28,6 \\
\hline
\end{tabular}

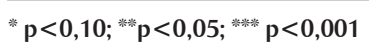

Tabela 5 Grau de satisfação dos trabalhadores acidentados entrevistados com o atendimento por instituição

\begin{tabular}{lcccc}
\hline \multirow{2}{*}{$\begin{array}{c}\text { Nota atribuída } \\
\text { ao tratamento }\end{array}$} & \multicolumn{3}{c}{ Instituição/financiamento } \\
& $N=108$ & $100 \%$ & $N=43$ & $100 \%$ \\
\hline Baixa $(0-7,0)$ & 34 & 31,5 & 9 & 20,9 \\
Média $(7,1-8,0)$ & 22 & 20,4 & 8 & 18,6 \\
Alta $(8,1-10)$ & 52 & 48,2 & 26 & 60,5 \\
\hline
\end{tabular}




\section{Discussão}

Verifica-se, com os dados deste estudo, que cerca de metade dos casos de acidentes de trabalho recebeu algum tipo de primeiros socorros, na maioria serviços de emergência, seguido de tratamento médico com duração média de 15 dias. A mais expressiva instituição financiadora ou prestadora do atendimento a esses casos foi o SUS. Verificou-se também que é muito pequena a participação das empresas empregadoras no financiamento do atendimento, salvo os custos com os planos de saúde para os trabalhadores, que não foram registrados na pesquisa. Embora reduzida, houve a participação dos trabalhadores no pagamento de atendimentos prestados por clínicas privadas, tanto por trabalhadores com carteira assinada como pelos informais. Houve uma concentração de pessoas com tratamento entre os acidentados mais jovens e para os casos classificados como “de trajeto". Ser jovem e sofrer acidente de trajeto foram categorias com maior proporção de casos mais graves, com a severidade baseada na duração do tratamento (resultados não apresentados). A maioria dos trabalhadores relatou alto nível de satisfação com o atendimento, seja no SUS, seja nas clínicas privadas, que apresentaram maior proporção de indivíduos com alta satisfação em comparação com os atendidos no SUS. Entre os trabalhadores informais, a proporção de alta satisfação com o tratamento foi também menor do que entre os trabalhadores com carteira $(\mathrm{p}<0,001)$.

Os resultados relativos à dimensão do uso dos serviços do SUS por trabalhadores acidentados, neste estudo, comparam-se aos encontrados por outras pesquisas de base populacional no Brasil (VILELA et al., 2001; CORDEIRO et al., 2005), embora sejam plausíveis diferenças na oferta e no acesso de serviços de saúde entre um estado do Nordeste e cidades do interior de São Paulo. Isso ocorre para todas as categorias sociais e tipo de vinculação previdenciária, revelando a importante responsabilidade do SUS na resposta às necessidades de saúde da população trabalhadora. Reforça a concepção da RENAST (DIAS \& HOEFEL, 2005), que assume a responsabilidade não só do atendimento médico pelo SUS, mas também a de organização da oferta de serviços de vigilância à saúde, com o diagnóstico dos problemas de saúde do trabalhador, e a proposição de medidas adequadas e viáveis de prevenção. Com isso, pode-se pensar, a partir da cobertura universal do SUS, superar a grande divisão entre segurados e não segurados em relação à prevenção de riscos ocupacionais, fundamental para reverter as tendências demonstradas de aumento da letalidade dos acidentes de trabalho (SANTANA et al., 2005). A natureza universalista do SUS ficou nítida nos resultados, que não revelaram diferenças no acesso ao tratamento referentes aos estratos sociais considerados, exceto aquelas que podem estar indicando gravidade do problema. Exceção foi o achado de maior proporção de não negros atendidos no SUS, o que precisa ser examinado com mais cuidado, pois pode ser resultante das múltiplas comparações realizadas.

A alta satisfação com o atendimento recebido não é surpreendente, considerando que outros estudos têm apresentado resultados semelhantes no Brasil (KOTAKA, PACHECO \& HIGAKI, 1997; KLOETZEL et al., 1998). Vale ressaltar que a comparação dos resultados especificamente para trabalhadores acidentados ficou limitada por não terem sido encontrados estudos específicos. Sabe-se que o SUS ainda passa por uma etapa de intensos investimentos na melhoria da qualidade da atenção, em especial na humanização do atendimento, em que pesem as conhecidas dificuldades do acesso (PAIM, 2002). Entretanto, a satisfação é elevada, o que deve ser visto com precaução, considerando que pode possivelmente expressar uma reação à gratuidade e à disponibilidade universal da assistência. Há que se observar que houve grande satisfação tanto por parte de trabalhadores de alto nível socioeconômico, quanto nos estratos pobres, sem diferenças expressivas do ponto de vista estatístico. Isso não ocorreu com trabalhadores informais, menos comumente satisfeitos que os contratados com carteira, algo a merecer investigações mais aprofundadas. Em um estudo realizado em Feira de Santana por Assis, Vilela e Nascimento (2003), de metodologia qualitativa, relatou-se uma tendência à avaliação negativa em relação aos serviços oferecidos pelo SUS por parte dos usuários. Resultados com alta satisfação como os encontrados neste estudo revelam que o SUS merece atenção especial na efetivação das políticas de proteção e segurança do trabalhador, na medida em que é, reconhecidamente, o principal serviço res- 
ponsável pela demanda de trabalhadores acidentados.

Como a pesquisa original não estava focalizada em padrões de utilização dos serviços, os dados são limitados, não se dispondo de informações sobre aspectos específicos do tratamento que poderiam ser empregadas para a melhoria de componentes do processo de funcionamento dos serviços. Todavia, permitem o conhecimento de algumas importantes características sobre o uso dos serviços de saúde e a satisfação com o tratamento, importantes especialmente pelo momento de implantação da RENAST, o qual deverá incorporar às responsabilidades do SUS a identificação dos riscos nos locais de trabalho e assim potencializar as ações de prevenção e não apenas de atendimento a casos já ocorridos

\section{Referências}

ASSIS, M. A. S.; VILLA, T. C. S.; NASCIMENTO, M. A. A. Acesso aos serviços de saúde: uma possibilidade a ser construída na prática. Ciênc. saúde coletiva, Rio de Janeiro, v. 8, n. 3, p. 815823, 2003.

BEDRIKOW, B. et al. Occupational health in Brazil. International archives of occupational and environmental health, Berlin, n. 70, p. 215-221, 1997.

BRASIL. Proposta de metodologia para elaboração da Agenda Nacional de Prioridades de Pesquisa e Desenvolvimento Tecnológico em Saúde. Brasília: Ministério da Saúde, 2002.

CONCEIÇÃO, P. S. A. et al. Acidentes de trabalho atendidos em serviço de emergência. Cad. Saúde Pública, Rio de Janeiro, v. 19, n. 1, p. 111-117, 2003.

CORDEIRO, R. C. et al. Underreporting of non-fatal work-related injuries in Brazil. Rev. Saúde Públ., São Paulo, v. 39, n. 2, p. 254-260, 2005.

DIAS, E. C.; HOEFEL, M. G. O desafio de implementar as ações de saúde do trabalhador no SUS: a estratégia da RENAST. Ciênc. saúde coletiva, Rio de Janeiro, v. 10, n. 4, p. 817-828, 2005.

HAGBERG, M. et al. Conceptual and definitional issues in occupational injury epidemiology. Am. J. Ind. Med., n. 32, p. 106-115, 1997.
(PENA \& COSTA, 2005). Espera-se que esta investigação inaugure uma série de estudos que tratem de informações que contribuam para uma mais ampla participação do SUS no atendimento das necessidades de saúde da população trabalhadora, voltando-se prioritariamente para a prevenção dos riscos ocupacionais e desse modo permitindo uma melhor condição de vida e bem-estar dos trabalhadores. A $8^{\text {a }}$ Conferência Nacional de Saúde estabeleceu como concepção de saúde a satisfação das necessidades básicas de acesso à alimentação regular e nutritiva, moradia adequada, transporte, educação e serviços de saúde eficientes. Portanto, o acesso a esses serviços não é apenas uma dimensão importante para o alcance da saúde e do bem-estar das pessoas, mas um direito de todos os cidadãos brasileiros.
KLOETZEL, K. et al. Quality control in primary health care. I consumer satisfaction. Cad. Saúde Pública, Rio de Janeiro, v. 14, n. 3, p. 263-268, 1998.

KOTAKA, F.; PACHECO, M. L. R.; HIGAKI, Y. The evaluation by users of hospitals which participate in the hospital quality program of S. Paulo state, Brazil. Rev. Saúde Pública, São Paulo, v. 31, n. 2, p. 171-177, 1997.

PAIM, J. Saúde, política e reforma sanitária. Salvador: ISC-CEPS, 2002.

PENA, P. G. L; COSTA, D. Persistem estratégias políticas ultraliberais para a saúde do trabalhador: uma contribuição ao debate. Ciênc. saúde coletiva, Rio de Janeiro, v. 10, n. 4, p. 808-815, 2005.

SANTANA V. S. et al. Acidentes de trabalho: custos previdenciários e dias perdidos de trabalho. Rev. Saúde Pública, São Paulo, v. 40, n. 6, p. 1004-1012, 2006.

SANTANA V. S. et al. Acidentes de trabalho não fatais em adolescentes. Cad. Saúde Pública, Rio de Janeiro, v. 19, n. 2, p. 407-420, 2003

SANTANA, V.S.; NOBRE L.; WALDVOGEL, B. Acidentes de trabalho no Brasil entre 1994 e 2004: uma revisão. Ciênc. saúde coletiva, Rio de Janeiro, v. 10, n. 4, p. 841-855, 2005.

TAKALA, J. Global estimates of fatal occupational injuries. Epidemiology, n. 10, p. 640-646, 1999 . 
VILELA, R. A. G.; RICARDI, G. V. F.;

IGUTI, A. M. Experiência do Programa de Saúde do Trabalhador de Piracicaba: desafios da vigilância em acidentes do trabalho. Informe Epidemiológico do SUS. São Paulo, v. 10, n. 2, p. 81-92, 2001. 